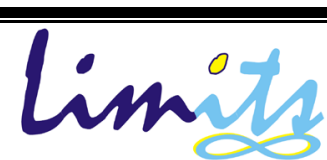

Limits: Journal of Mathematics and Its Applications

E-ISSN: 2579-8936

P-ISSN: 1829-605X

Vol. 18, No. 1, Mei 2021, 37-44

DOI: http://dx.doi.org/10.12962/limits.v18i1.8291

\title{
The half-Space Model Problem for Compressible Fluid Flow
}

\author{
Sri Maryani ${ }^{1}$ *, Lukman B Nugroho ${ }^{2}$, Agus Sugandha ${ }^{3}$, Bambang H Guswanto ${ }^{4}$ \\ $1,2,3,4$ Departement of Matematika Jenderal Soedirman University, Indonesia \\ e-mail:sri.maryani@unsoed.ac.id
}

Diajukan: 30 Desember 2020, Diperbaiki: 31 Maret 2021, Diterima: 21 April 2021

\begin{abstract}
In this paper we consider the solution formula for Stokes equation system without surface tension in half-space. More precisely, we deal with the solution of velocity and density for the model problem. This result is the basic step to estimate the solution operator of the model problem. We investigate the solution operator for the model problem in $N$-Dimensional Euclidean space $(\mathbb{N} \geq 2)$
\end{abstract}

Keywords: Stokes equation, half-space, $N$-Dimensional Euclidean space, surface tension.

\section{Introduction}

Fluid and flows are pervading our daily lifes without our conscious perception. For examples the air we breathe, the shower in the bathroom with the shampoo, coffee or tea, the blood in our vascular tree including the heart and the brain, the ingredients of food like mayonnaise, oil, vinegar, yogurt, etc. The design and the engineering of new material like polymers, plastics, ceramics, foam, etc have produced complex fluids because the material processing based on extrusion, molding, blowing etc., is using them in the fluid state.

Studying about fluid motion is very interesting point in fluid dynamics. Recently, there has been increasing amount of literature on fluid flow. Many researchers investigated about this material. However, many of them conducted in numerical analysis and rarely of them investigated fluid motion in the mathematical analysis approach. In fluid mechanics, the flow of incompressible fluid which is described as Navier-Stokes equation (NSE). Meanwhile, in mathematics, NSE can be written in the partial differential equations. NSE is firstly introduced by Swiss mathematician Leonhard Euler in $18^{\text {th }}$ century. He investigated the flow of the incompressible and frictionless fluids. The linearize of the NSE we known as Stokes equations.

Historically, as far as we know that the Stokes flow is the linearized form of the NavierStokes equations in the limit of small Reynold number. Stokes' formula is the basis method to measure the unit charge. The pioneer using this formula is Millikan to measure the charge on the electron [1]. In these experiments fine droplets produced by an oil spray were placed in the space 
between horizontal plates forming a plane capacitor. The droplets have a charge owing to electrification in the spraying process or absorption of ions from the air. By observing under a microscope the rate of fall of a droplet by the effect of its weight alone, we can use Stokes' formula to calculate the radius and hence the mass of the drop (whose density is known). Then, by applying a suitable potential difference across the capacitor, we can bring the droplet to rest, the downward force of gravity being balanced by the upward electrical force on the charged droplet. Knowing the weight of the droplet and the electric field strength, we can calculate the charge on the droplet. Such measurements show that the charge on the droplets is always an integral multiple of a certain quantity, which is evidently the unit charge.

Several recent studies investigating this model problem have been carried out not only on to find the solution formula but also to estimate the operator solution families of the model problem. In 2015 Murata [2] has been investigated the Stokes equation with slip boundary condition. She investigated not only the local well-posedness of the model problem, but also global wellposedness. The regularity of the model problem approached using the $\mathcal{R}$-boundedness of the solution operator.

Afterward, Maryani [3] concerned to study the compressible fluid motion for the OldroydB Model. She investigated the local well-posedness of the non-Newtonian compressible barotropic flow in the maximal $L_{p}-L_{q}$ regularity class in bounded and unbounded domain. Meanwhile, Maryani and Saito [4] studied the $\mathcal{R}$-boundedness of the solution operator families for two phase problem of Stokes equation in half-space. On 2020 Inna et.al [5] considered the half-space model problem for the compressible fluid motion of the Korteweg type.

In this paper we consider the solution formula of the Stokes equation in half-space without surface tension using Fourier transform. As we know that the Stokes formula is usually used to determine the viscosity of a liquid or gas from a measurement of the rate of fall of a solid sphere in it . The viscosity may also be assigned by means of Poiseuille's formula, by measuring the rate of outflow of a liquid from a pipe along which it is impelled by a given pressure difference.

To introduce our main result, first of all we introduce the notation. For a scalar-valued function $v=v(x)$ and vector-valued function $\mathbf{v}=\mathbf{v}(\mathrm{x})=\left\langle\mathrm{v}_{1}(\mathrm{x}), \ldots, \mathrm{v}_{\mathrm{N}}(\mathrm{x})\right\rangle^{\mathrm{T}}$, we set for $\partial_{\mathrm{k}}=$ $\frac{\partial}{\partial \mathrm{x}_{\mathrm{k}}},(\mathrm{k}=1, \cdots, \mathrm{N})$

$$
\begin{gathered}
\nabla \mathrm{u}=\left(\partial_{1} \mathrm{u}, \ldots, \partial_{\mathrm{N}} \mathrm{u}\right)^{\mathrm{T}}, \quad \Delta \mathrm{u}=\sum_{\mathrm{k}=1}^{\mathrm{N}} \partial_{\mathrm{k}}^{2} \mathrm{u}, \quad \Delta \mathrm{v}=\left\langle\Delta \mathrm{v}_{1}, \ldots, \Delta \mathrm{v}_{\mathrm{N}}\right\rangle^{\mathrm{T}}, \\
\operatorname{div} \mathrm{v}=\sum_{\mathrm{k}=1}^{\mathrm{N}} \partial_{\mathrm{k}} \mathrm{v}_{\mathrm{k}}, \quad \nabla \mathrm{v}=\left\{\partial_{\mathrm{k}} \mathrm{v}_{\ell} \mid \mathrm{k}, \ell=1,2,3, \ldots, \mathrm{N}\right\}, \quad \nabla^{2} \mathbf{v} \\
=\left\{\partial_{\mathrm{k}} \partial_{\ell} \mathrm{v}_{\mathrm{m}} \mid \mathrm{k}, \ell, \mathrm{m}=1,2,3, \ldots, \mathrm{N}\right\} .
\end{gathered}
$$

The set of all natural number is denoted by $\mathbb{N}$ and $\mathbb{N}_{0}=\mathbb{N} \cup\{0\}$. Let $\mathcal{F}_{x}=\mathcal{F}$ and $\mathcal{F}_{\xi}^{-1}=$ 
$\mathcal{F}^{-1}$ denote the Fourier transform and the Fourier inverse transform, which defined by

$$
\mathcal{F}_{x}[f](\xi)=\int_{\mathbb{R}^{N}} e^{-i x \cdot \xi} f(x) d x, \quad \mathcal{F}_{\xi}^{-1}[g](x)=\frac{1}{(2 \pi)^{N}} \int_{\mathbb{R}^{N}} e^{i x \cdot \xi} g(\xi) d \xi,
$$

respectively. We also write $\hat{f}(\xi)=\mathcal{F}_{x}[f](\xi)$. Let $\mathcal{L}$ and $\mathcal{L}^{-1}$ the denote the Laplace transform and the Laplace inverse transform, which defined by

$$
\begin{gathered}
\mathcal{L}[f](\lambda)=\int_{-\infty}^{\infty} e^{-\lambda t} f(t) d t=\mathcal{F}_{t}\left[e^{-\gamma t} f(t)\right](\tau), \\
\mathcal{L}^{-1}[g](t)=\frac{1}{2 \pi} \int_{-\infty}^{\infty} e^{\lambda t} g(\tau) d \tau=e^{\gamma t} \mathcal{F}_{t}^{-1}[g(\tau)](t),
\end{gathered}
$$

with $\lambda=\gamma+i \tau \in \mathbb{C}$, respectively.

\section{Literature Review}

Let $\mathbf{u}$ and $\rho$ be the velocity and density field, respectively. We consider the Stokes equation system without surface tension in bounded domain in half-space. We define $\mathbb{R}_{+}^{N}$ and $\mathbb{R}_{0}^{N}(\mathbb{N} \geq 2)$ be the half-space and its boundary, respectively by

$$
\begin{aligned}
& \mathbb{R}_{+}^{N}=\left\{\mathbf{x}=\left(\mathrm{x}_{1}, \mathrm{x}_{2}, \ldots, \mathrm{x}_{3}\right) \in \mathbb{R}^{\mathrm{N}} \mid \mathrm{x}_{N}>0\right\}, \text { and } \\
& \mathbb{R}_{0}^{N}=\left\{\mathbf{x}=\left(\mathrm{x}_{1}, \mathrm{x}_{2}, \ldots, \mathrm{x}_{3}\right) \in \mathbb{R}^{\mathrm{N}} \mid \mathrm{x}_{N}=0\right\} .
\end{aligned}
$$

The resolvent problem of Stokes equations are being described by the set of equations,

$$
\left\{\begin{array}{l}
\lambda \rho+\gamma \operatorname{div} \mathbf{u}=f \quad \text { in } \mathbb{R}_{+}^{N} \\
\lambda \mathbf{u}-\operatorname{Div} \mathbf{S}(\mathbf{u}, \rho)=\mathbf{g} \text { in } \mathbb{R}_{+}^{N} \\
\mathbf{S}(\mathbf{u}, \rho) \mathbf{n}=\mathrm{h} \quad \text { on } \mathbb{R}_{0}^{N}
\end{array},\right.
$$

where $f, \mathbf{g}$ and $\mathrm{h}$ are scalar vector and $\mathbf{S}(\mathbf{u}, \rho)$ is the stress tensor which is defined by

$$
\mathbf{S}(\mathbf{u}, \rho)=2 \alpha \mathbf{D}(\mathbf{u})+(\beta \operatorname{div} \mathbf{u}-\gamma \rho) \mathbf{I},
$$

and $\mathbf{n}=(0,0, \ldots,-1)$ stands for the unit outer normal to $\mathbb{R}_{+}^{N}$. The doubled deformation $\mathbf{D}(\mathbf{u})$ tensor whose $(i, j)$ components are $\mathbf{D}_{i j}(\mathbf{u})=\partial_{\mathrm{i}} u_{j}+\partial_{j} u_{i}\left(\partial_{i}=\partial / \partial x_{i}\right)$, I the $N \times N$ identity matrix, $\alpha, \beta$ and $\gamma$ are positive constants and also $\operatorname{div} \mathbf{u}=\sum_{j=1}^{N} \partial_{j} u_{j}$.

Before we state the main result, first of all we introduce the definition of sobolev space $W_{p}^{m}(\Omega)$ is defined by

$$
W_{p}^{k, m}(\Omega)=\left\{(\rho, \mathbf{u}) \mid \rho \in W_{p}^{k}(\Omega), \mathbf{u} \in W_{p}^{m}(\Omega)\right\} .
$$

Definition 1 (Adams and Fournier, [6])

Let $\mathrm{k} \in \mathbb{N} \cup\{0\}$ and $\mathrm{p} \in[1, \infty)$ then the Sobolev Space $\mathrm{W}_{\mathrm{q}}^{\mathrm{m}}(\Omega)$ is defined by

$$
\mathrm{W}_{\mathrm{q}}^{\mathrm{m}}(\Omega):=\left\{\mathbf{u} \in \mathrm{L}_{\mathrm{q}}(\Omega) \mid \mathrm{D}^{\alpha} \mathbf{u} \in \mathrm{L}_{\mathrm{q}}(\Omega), \forall \alpha \text { with }|\alpha| \leq \mathrm{m}\right\} .
$$

Furthermore, we state the main theorem of this paper

Theorem 2. Let $N<q<\infty, 2<p<\infty$ then there exists Lopatinski matrix $L$ i.e 


$$
L=\left(\begin{array}{ll}
L_{11} & L_{12} \\
L_{21} & L_{22}
\end{array}\right)
$$

with

$$
\begin{gathered}
L_{11}=\alpha B-\left(\alpha+\left|\xi^{\prime}\right|\right) A, L_{12}=2 A B\left(\alpha+\left|\xi^{\prime}\right|^{2}\right)+\left|\xi^{\prime}\right| \\
L_{21}=-\frac{(2 \alpha+\beta+\delta) A^{2}+2(\beta+\delta)\left|\xi^{\prime}\right|^{2}}{\left|\xi^{\prime}\right|^{2}}, L_{22}=\frac{(2 \alpha+\beta+\delta)\left(2 A^{2} B+B\left|\xi^{\prime}\right|^{2}\right)-2(\beta+\delta) B\left|\xi^{\prime}\right|^{2}}{\left|\xi^{\prime}\right|^{2}}
\end{gathered}
$$

and

$$
\operatorname{det} L=L_{11} L_{22}-L_{12} L_{21} \text {. }
$$

Then problem (2) admits a unique solution formula of $(\rho, \mathbf{u}) \in W_{p}^{1,2}(\Omega)$ with

$$
\begin{aligned}
& \hat{u}_{j}\left(\xi^{\prime}, x_{N}\right)= \sum_{k=1}^{N-1} \frac{\eta\left(i \xi_{j}\right)\left(i \xi_{k}\right)\left(L_{22}+2 B L_{21}\right)}{B(B+A) \operatorname{det} L} \cdot \frac{\left|\xi^{\prime}\right|^{2}+A}{\left|\xi^{\prime}\right|^{2}}\left(\frac{-B e^{-A x_{N}}+2 A e^{-B x_{N}}}{(B-A)}\right) \widehat{h_{k}}\left(\xi^{\prime}, 0\right) \\
&+\frac{\eta\left(i \xi_{j}\right)\left(L_{12}+2 B L_{11}\right)}{B(B+A) \operatorname{det} L} \cdot \frac{\left|\xi^{\prime}\right|^{2}+A}{\left|\xi^{\prime}\right|^{2}}\left(\frac{B e^{-A x_{N}}-2 A e^{-B x_{N}}}{(B-A)}\right) \widehat{h_{N}}\left(\xi^{\prime}, 0\right) \\
&-\sum_{k=1}^{N-1} \frac{\left(i \xi_{j}\right)\left(i \xi_{k}\right) L_{21}}{\operatorname{det} L} e^{-B x_{N} \widehat{h_{k}}}\left(\xi^{\prime}, 0\right)+\frac{\left(i \xi_{j}\right) L_{11}}{\operatorname{det} L} e^{-B x_{N} \widehat{h_{N}}\left(\xi^{\prime}, 0\right)} \\
&+\frac{1}{\alpha B} e^{-B x_{N} \widehat{h_{k}}\left(\xi^{\prime}, 0\right)}
\end{aligned}
$$

for $j=1, \ldots, N-1$ and

$$
\begin{aligned}
\hat{u}_{N}\left(\xi^{\prime}, x_{N}\right)= & \sum_{k=1}^{N-1} \frac{\left(i \xi_{k}\right)}{\operatorname{det} L} \cdot\left(\frac{\eta\left(L_{22}+2 B L_{21}\right) A}{\left(B^{2}-A^{2}\right)} \cdot \frac{\left|\xi^{\prime}\right|^{2}+A}{\left|\xi^{\prime}\right|^{2}} e^{-A x_{N}}-L_{21} e^{-B x_{N}}\right) \widehat{h_{k}}\left(\xi^{\prime}, 0\right) \\
& -\frac{1}{\operatorname{det} L}\left(\frac{\eta A\left(L_{12}+2 B L_{11}\right)}{\left(B^{2}-A^{2}\right)} \cdot \frac{\left|\xi^{\prime}\right|^{2}+A}{\left|\xi^{\prime}\right|^{2}} e^{-A x_{N}}-L_{11} e^{-B x_{N}}\right) \widehat{h_{N}}\left(\xi^{\prime}, 0\right)
\end{aligned}
$$

with $A^{2}=\left|\xi^{\prime}\right|^{2}+\alpha^{-1} \lambda, B^{2}=\left|\xi^{\prime}\right|^{2}+(2 \alpha+\beta+\delta)^{-1} \lambda$ and $\delta=\gamma^{2} \lambda^{-1}$.

\section{Research Methodology}

In this research methodology, we use literature review of the related articles, especially [7]. In this paper, we put the different approach of the general solution of velocity as in [7]. This research focus on considering the solution formula of the compressible Stokes equation system (2) without surface tension by using Fourier transform. First of all we construct the compressible Stokes equation without surface tension in half-space case. Then, by applying the Fourier transformation to model problem, we get new equation system. Finally, the solution formula of the model problem are furnished. 


\section{$4 \quad$ Research and Discussion}

\section{Reduce System}

In this section, we formulated the model problem (2) and state our main result. Let $\mathbb{R}_{+}^{N}$ and $\mathbb{R}_{0}^{N}$ as defined in (1). We consider for $\lambda \neq 0$, inserting $\rho=\lambda^{-1}(f-\gamma \operatorname{div} \mathbf{u})$ to the second and third equation of (2), then we have

$$
\begin{cases}\lambda \mathbf{u}-\alpha \Delta \mathbf{u}-(\alpha+\beta+\delta) \nabla \operatorname{div} \mathbf{u}=\mathbf{F} & \text { in } \mathbb{R}_{+}^{N} \\ \alpha\left(\partial_{\mathrm{j}} \mathrm{u}_{\mathrm{N}}+\partial_{\mathrm{N}} \mathrm{u}_{\mathrm{j}}\right)=-\mathrm{h}_{\mathrm{j}} & \text { on } \mathbb{R}_{0}^{N} \\ 2 \alpha \partial_{\mathrm{N}} \mathrm{u}_{\mathrm{N}}+(\beta+\delta) \operatorname{div} \mathbf{u}=-\mathrm{h}_{\mathrm{N}} & \text { on } \mathbb{R}_{0}^{N}\end{cases}
$$

for $j=1, \ldots, N-1$ and $\mathbf{F}=\mathbf{g}-\lambda^{-1} \gamma \nabla f$.

Moreover, we derive a solution formula of (5). For this purpose, applying the partial Fourier transform to (5) i.e

$$
\begin{gathered}
\hat{u}=\hat{u}\left(x_{N}\right)=\hat{u}\left(\xi^{\prime}, x_{N}\right)=\int_{\mathbb{R}^{N-1}} e^{-i x^{\prime} \cdot \xi^{\prime}} u\left(x^{\prime}, x_{N}\right) d x^{\prime} \\
\mathcal{F}_{\xi}^{-1}\left[\hat{u}\left(\xi^{\prime}, x_{N}\right)\right]\left(x^{\prime}\right)=\frac{1}{(2 \pi)^{N-1}} \int_{\mathbb{R}^{N-1}} e^{i x \prime \cdot \xi^{\prime}} \hat{u}\left(\xi^{\prime}, x_{N}\right) d \xi^{\prime},
\end{gathered}
$$

where $\xi^{\prime}=\left(\xi_{1}, \ldots, \xi_{N-1}\right) \in \mathbb{R}^{N-1}$, we have

$$
\left\{\begin{array}{c}
\alpha\left(\alpha^{-1} \lambda+\left|\xi^{\prime}\right|^{2}\right) \hat{u}_{j}-\alpha \partial_{N}^{2} \hat{u}_{j}+(\alpha+\beta+\delta) i \xi_{j}\left(i \xi^{\prime} \cdot \hat{u}^{\prime}+\partial_{N} \hat{u}_{N}\right)=0\left(x_{N}>0\right) \\
\alpha\left(\alpha^{-1} \lambda+\left|\xi^{\prime}\right|^{2}\right) \hat{u}_{N}-\alpha \partial_{N}^{2} \hat{u}_{N}+(\alpha+\beta+\delta) \partial_{N}\left(i \xi^{\prime} \cdot \hat{u}^{\prime}+\partial_{N} \hat{u}_{N}\right)=0\left(x_{N}>0\right) \\
\left.\alpha\left(i \xi_{j} \hat{u}_{N}+\partial_{N} \hat{u}_{N}\right)\right|_{x_{N}=0}=-\widehat{h}_{j}\left(\xi^{\prime}, 0\right) \\
2 \alpha \partial_{N} u_{N}+\left.(\beta+\delta)\left(i \xi^{\prime} \cdot \hat{u}^{\prime}+\partial_{N} \hat{u}_{N}\right)\right|_{x_{N}=0}=-\widehat{h_{N}}\left(\xi^{\prime}, 0\right)
\end{array},\right.
$$

for $j=1, \ldots, N-1$ and $i \xi^{\prime} \cdot \hat{u}^{\prime}=\sum_{k=1}^{N-1} i \xi_{k} u_{k}$.

Let $\widehat{\mathbf{u}}=\left(\hat{u}_{1}, \ldots, \hat{u}_{N}\right)$ have general formula in the following

$$
\hat{u}_{\ell}=P_{\ell} e^{-A x_{N}}+Q_{\ell} e^{-B x_{N}},
$$

then we get

$$
\begin{gathered}
\partial_{N} \hat{u}_{N}=-A P_{N} e^{-A x_{N}}-B P_{N} e^{-B x_{N}}, \\
\partial_{N}^{2} \hat{u}_{N}=A^{2} P_{N} e^{-A x_{N}}+B^{2} P_{N} e^{-B x_{N}}, \\
i \xi^{\prime} \cdot \hat{u}^{\prime}=\sum_{k=1}^{N-1} i \xi_{j}\left(P_{j} e^{-A x_{N}}+Q_{j} e^{-B x_{N}}\right) .
\end{gathered}
$$

Applying div to first equation of (5) we have

$$
(\lambda-(2 \alpha+\beta+\delta) \Delta) \operatorname{div} \mathbf{u}=0 .
$$

Multiplying (5) by $(\lambda-(2 \alpha+\beta+\delta) \Delta)$ then using (11), we have

$$
(\lambda-\alpha \Delta)(\lambda-(2 \alpha+\beta+\delta) \Delta) \mathbf{u}=0 .
$$


Applying Fourier transform to (12), we have formula

$$
A^{2}=\left|\xi^{\prime}\right|^{2}+(2 \alpha+\beta+\delta)^{-1} \lambda \text { and } B^{2}=\left|\xi^{\prime}\right|^{2}+\alpha^{-1} \lambda .
$$

Substituting (7), (8), (9) and (10) to equation system of (6) and equating the coefficients of $e^{-A x_{N}}$ and $e^{-B x_{N}}$, we have new equation system

$$
\left\{\begin{array}{c}
\alpha\left(B^{2}-A^{2}\right) P_{j}-(\alpha+\beta+\delta) i \xi_{j}\left(i \xi^{\prime} \cdot P^{\prime}-A P_{N}\right)=0 \\
\alpha\left(B^{2}-A^{2}\right) P_{N}+(\alpha+\beta+\delta) A\left(i \xi^{\prime} \cdot P^{\prime}-A P_{N}\right)=0 \\
i \xi^{\prime} \cdot P^{\prime}+i \xi^{\prime} \cdot Q^{\prime}-2 B Q_{N}=0 \\
\alpha\left(A P_{j}+B Q_{j}-i \xi_{j}\left(P_{N}+Q_{N}\right)\right)=\widehat{h}_{J}\left(\xi^{\prime}, 0\right) \\
(2 \alpha+\beta+\delta)\left(A P_{N}+B Q_{N}\right)-(\beta+\delta)\left(i \xi^{\prime} \cdot P^{\prime}+i \xi^{\prime} \cdot Q^{\prime}\right)=\widehat{h_{N}}\left(\xi^{\prime}, 0\right) .
\end{array}\right.
$$

Let $\left(i \xi^{\prime} \cdot P^{\prime}-A P_{N}\right)=K$, so that from first and second equation of (14), we have the formula of $P_{j}$ and $P_{N}$

$$
P_{j}=\frac{(\alpha+\beta+\delta) i \xi_{j}}{\alpha\left(B^{2}-A^{2}\right)} K \text { and } P_{N}=-\frac{(\alpha+\beta+\delta) A}{\alpha\left(B^{2}-A^{2}\right)} K
$$

respectively.

Therefore, we have the new solution formula of $K$,

$$
K=\frac{\alpha\left(B^{2}-A^{2}\right)}{(\alpha+\beta+\delta)|\xi|^{2}}\left(i \xi^{\prime} \cdot Q^{\prime}-2 B Q_{N}\right) .
$$

By using equation (16), we can find the new formula of $P_{j}$ and $P_{N}$

$$
P_{j}=\frac{i \xi_{j}}{\left|\xi^{\prime}\right|^{2}}\left(i \xi^{\prime} \cdot Q^{\prime}-2 B Q_{N}\right) \text { and } P_{N}=-\frac{A}{\left|\xi^{\prime}\right|^{2}}\left(i \xi^{\prime} \cdot Q^{\prime}-2 B Q_{N}\right) \text {, }
$$

respectively. Multiplying both side of the fourth equation of (14) by $\sum_{j=1}^{N-1} i \xi_{j}$, we have

$$
\begin{gathered}
\alpha\left(A \sum_{j=1}^{N-1} i \xi_{j} P_{j}+B \sum_{j=1}^{N-1} i \xi_{j} Q_{j}-\sum_{j=1}^{N-1} i \xi_{j} i \xi_{j}\left(P_{N}+Q_{N}\right)\right)=\sum_{j=1}^{N-1} i \xi_{j} \widehat{h}_{j}\left(\xi^{\prime}, 0\right) \\
\alpha\left(A i \xi^{\prime} \cdot P^{\prime}+B i \xi^{\prime} \cdot Q^{\prime}-|\xi|^{2}\left(P_{N}+Q_{N}\right)\right)=i \xi^{\prime} \cdot \widehat{h^{\prime}}\left(\xi^{\prime}, 0\right) .
\end{gathered}
$$

Combining equation (18) with last equation of (14) and (17), we have a linear system

$$
L\left(\begin{array}{c}
i \xi^{\prime} \cdot Q^{\prime} \\
Q_{N}
\end{array}\right)=\left(\begin{array}{c}
i \xi^{\prime} \cdot \widehat{h^{\prime}}\left(\xi^{\prime}, 0\right) \\
\widehat{h_{N}}\left(\xi^{\prime}, 0\right)
\end{array}\right),
$$

with $L$ adalah $2 \times 2$ of Matrix Lopatinski with entries are

$$
\begin{gathered}
L_{11}=\alpha B-\left(\alpha+\left|\xi^{\prime}\right|\right) A, L_{12}=2 A B\left(\alpha+\left|\xi^{\prime}\right|^{2}\right)+\left|\xi^{\prime}\right| \\
L_{21}=-\frac{(2 \alpha+\beta+\delta) A^{2}+2(\beta+\delta)\left|\xi^{\prime}\right|^{2}}{\left|\xi^{\prime}\right|^{2}}, L_{22}=\frac{(2 \alpha+\beta+\delta)\left(2 A^{2} B+B\left|\xi^{\prime}\right|^{2}\right)-2(\beta+\delta) B\left|\xi^{\prime}\right|^{2}}{\left|\xi^{\prime}\right|^{2}} .
\end{gathered}
$$

By using Cramer's rule, from (19), we obtain the formula of $i \xi^{\prime} \cdot Q^{\prime}$ and $Q_{N}$,

$$
i \xi^{\prime} \cdot Q^{\prime}=\frac{L_{22} i \xi^{\prime} \cdot \hat{h}\left(\xi^{\prime}, 0\right)-L_{12} \widehat{h_{N}}\left(\xi^{\prime}, 0\right)}{\operatorname{det} L}
$$


and

$$
Q_{N}=\frac{L_{11} \widehat{h_{N}}\left(\xi^{\prime}, 0\right)-L_{21} i \xi^{\prime} \cdot \widehat{h}\left(\xi^{\prime}, 0\right)}{\operatorname{det} L}
$$

respectively, with

$$
\operatorname{det} L=L_{11} L_{22}-L_{12} L_{21}
$$

formula $L_{11}, L_{12}, L_{21}$ and $L_{22}$ as in (20). Moreover, we have

$$
\begin{aligned}
P_{j} & =-\frac{\eta\left(i \xi_{j}\right)\left(\left|\xi^{\prime}\right|^{2}+A\right)}{\left(B^{2}-A^{2}\right)\left|\xi^{\prime}\right|^{2} \operatorname{det} L}\left\{\left(L_{22}+2 B L_{21}\right) i \xi^{\prime} \cdot \hat{h}\left(\xi^{\prime}, 0\right)-\left(L_{12}+2 B L_{11}\right) \widehat{h_{N}}\left(\xi^{\prime}, 0\right)\right\}, \\
P_{N} & =\frac{\eta\left(i \xi_{j}\right) A\left(\left|\xi^{\prime}\right|^{2}+A\right)}{\left(B^{2}-A^{2}\right)\left|\xi^{\prime}\right|^{2} \operatorname{det} L}\left\{\left(L_{22}+2 B L_{21}\right) i \xi^{\prime} \cdot \hat{h}\left(\xi^{\prime}, 0\right)-\left(L_{12}+2 B L_{11}\right) \widehat{h_{N}}\left(\xi^{\prime}, 0\right)\right\}, \\
Q_{j} & =\frac{\left(i \xi_{j}\right)}{\operatorname{det} L}\left(\frac{2 \eta A\left(\left|\xi^{\prime}\right|^{2}+A\right)}{\left(B^{2}-A^{2}\right)\left|\xi^{\prime}\right|^{2}}\left(L_{22}+2 B L_{21}\right)-L_{21}\right) i \xi^{\prime} \cdot \hat{h}\left(\xi^{\prime}, 0\right)+\frac{1}{\alpha B} \widehat{h_{j}}\left(\xi^{\prime}, 0\right), \\
& =\frac{\left(i \xi_{j}\right)}{\operatorname{det} L}\left(\frac{2 \eta A\left(\left|\xi^{\prime}\right|^{2}+A\right)}{\left(B^{2}-A^{2}\right)\left|\xi^{\prime}\right|^{2}}\left(L_{22}+2 B L_{21}\right)-L_{21}\right) \widehat{h_{N}}\left(\xi^{\prime}, 0\right), \\
Q_{N} & =\frac{L_{11} \widehat{h_{N}}\left(\xi^{\prime}, 0\right)-L_{21} i \xi^{\prime} \cdot \widehat{h}\left(\xi^{\prime}, 0\right)}{\operatorname{det} L}
\end{aligned}
$$

where

$$
\eta=\frac{\alpha+\beta+\delta}{\alpha} \text {. }
$$

Substituting (21), (22), (23), (24) and (25) to (7), we obtain the formula of $\widehat{\mathbf{u}}=\hat{u}_{j}=$ $\left\langle\hat{u}_{1}, \hat{u}_{2}, \ldots, \hat{u}_{N-1}\right\rangle$ and $\hat{u}_{N}$ in equation system of (5) which completes the proof of Theorem 2

\section{Conclusion}

The conclusion of the article that we can find the solution formula of velocity $\widehat{\mathbf{u}}$ and density $\rho$ of the model problem (2). We can see that for the further research, we can estimate the operator families of the solution.

\section{Acknowledgements}

The authors would like to thank Jenderal Soedirman University (UNSOED). The first author was supported by BLU UNSOED research scheme of International Research Collaboration (IRC) contract number: T/176/UN23.18/PT.01.03/2020.

\section{References}

[1] A. I. Akhiezer, I. A. Akhiezer, and R. V. Polovin, Plasma Electrodynamics: Linear Theory, vol. 1. Elsevier, 2017. 
[2] M. Murata, "On a maximal Lp-Lq approach to the compressible viscous fluid flow with slip boundary condition," Nonlinear Anal. Theory, Methods Appl., vol. 106, pp. 86-109, 2014.

[3] S. Maryani, "On the free boundary problem for the Oldroyd-B Model in the maximal LpLq regularity class,” Nonlinear Anal. Theory, Methods Appl., vol. 141, pp. 109-129, 2016.

[4] S. Maryani and H. Saito, "On the $\$ \backslash$ mathcal $\{R\} \$$-boundedness of solution operator families for two-phase Stokes resolvent equations," Differ. Integr. Equations, vol. 30, no. 1/2, pp. 1-52, 2017.

[5] S. Inna, S. Maryani, and H. Saito, "Half-space model problem for a compressible fluid model of Korteweg type with slip boundary condition," in Journal of Physics: Conference Series, 2020, vol. 1494, no. 1, p. 12014.

[6] R. A. Adams and J. J. F. Fournier, Sobolev spaces. Elsevier, 2003.

[7] D. Götz and Y. Shibata, "On the $\mathscr{R}$-boundedness of the solution operators in the study of the compressible viscous fluid flow with free boundary conditions," Asymptot. Anal., vol. 90, no. 3-4, pp. 207-236, 2014. 